\title{
A reusable, locally manufactured, half- face respirator provides better protection than fitted disposable N95 masks: development and quantitative fit-testing comparison
}

Arnaud Romeo Mbadjeu Hondjeu

University Health Network

William C. K. Ng ( $\sim$ drwilliam.ng@mail.utoronto.ca )

University of Toronto

Vahid Anwari

University Health Network

Maggie Z.X. Xiao

University of Alberta

Dmitry Rozenberg

University Health Network

Kate Kazlovich

University of Toronto

Edem Andy Afenu

University of Toronto

Joshua Qua Hiansen

University Health Network

Azad Mashari

University Health Network

Research Article

Keywords: COVID-19, reusable respirator, QNFT

Posted Date: May 14th, 2021

DOl: https://doi.org/10.21203/rs.3.rs-456096/v1

License: (c) (1) This work is licensed under a Creative Commons Attribution 4.0 International License.

Read Full License 


\title{
A reusable, locally manufactured, half-face respirator provides better protection than fitted disposable N95 masks: development and quantitative fit-testing comparison
}

\author{
Arnaud Romeo Mbadjeu Hondjeu ${ }^{1,2}$ William C. K. Ng ${ }^{3,4,5 \star}$, Vahid Anwari ${ }^{1,6}$, Maggie Z.X. Xiao \\ Dmitry Rozenberg ${ }^{8,9}$, Kate Kazlovich ${ }^{1,10}$, Edem Andy Afenu ${ }^{9,10}$, Joshua Qua Hiansen ${ }^{1}$, Azad \\ Mashari ${ }^{1,3,4^{*}}$
}

1 The Lynn \& Arnold Irwin Advanced Perioperative Imaging Lab, Toronto General Hospital, University Health Network, Toronto, ON, Canada

2 Department of Anesthesiology and Pain Management, Toronto Western Hospital, University Health Network, Toronto, Ontario, Canada

3 Department of Anesthesiology and Pain Management, Toronto General Hospital, University Health Network, Toronto, Ontario, Canada

4 Department of Anesthesiology and Pain Medicine, University of Toronto, Toronto, Ontario, Canada, 5 Department of Anesthesiology and Pain Management, Cardiac Division, Hospital for Sick Children, Toronto, Ontario, Canada,

6 Joint Department of Medical Imaging, Toronto General Hospital, University Health Network, Toronto, ON, Canada

7 Faculty of Medicine, University of Alberta, Edmonton, Alberta, Canada,

8 Division of Respirology and Lung Transplantation, Toronto General Hospital Research Institute, University Health Network, Toronto, Ontario, Canada, 9 Department of Medicine, University of Toronto, Toronto, Ontario, Canada

10 Institute of Biomaterials and Biomedical Engineering University of Toronto, Toronto, Ontario, Canada

* Corresponding authors: William C. K. Ng (William.Ng@uhn.ca), Azad Mashari (Azad.Mashari@uhn.ca) 


\begin{abstract}
The COVID-19 pandemic has led to persistent supply shortages of respiratory protective equipment in many jurisdictions. Reusable industrial respirators have been proposed and deployed as an alternative, but also face severe supply limitations. In addition, industrial respirators do not filter the user's expired breath, a major limitation in health care settings where bidirectional protection is required. We present the development and quantitative testing of a reusable silicone respirator that can be locally manufactured using low-cost desktop infrastructure. Using standardized quantitative fit-testing (QNFT including resting and activity components according to CSA Z94.4-18) in a cohort of 41 healthcare workers (HCWs), we compared the performance of the mask to the individually-fitted disposable N95 masks that the HCWs had been assigned by our institution. Overall QNFT pass rates for disposable N95 respirators were $58.5 \%$ vs. $100 \%$ for the reusable mask. For a production run of 1000 masks, unit cost is approximately $\$ 25 \mathrm{CAD}$ in materials and 35 minutes in labor per mask. The device requires further testing to assess flow resistance, carbon dioxide rebreathing, and full conformance with technical standards required for regulatory approval.
\end{abstract}




\section{Introduction}

Health care workers (HCWs) face hazardous occupational exposures to infectious organisms, many of which are spread through airborne or aerosolized droplet routes ${ }^{1}$. To reduce the risk of infection, the Centers for Disease Control and Prevention (CDC) recommends the use of personal protective equipment (PPE): gowns, gloves, face shields, goggles and a disposable N95 respirator.

N95 is a standard defined by the United States' National Institute for Occupational Safety and Health $(\mathrm{NIOSH})$ requiring an approved device to remove at least $95 \%$ of airborne particles of $\sim 0.3 \mu \mathrm{m}$ from inspired air with a complete face seal ${ }^{2}$. Comparable standards from other jurisdictions, such as FFP in Europe, KN95 in China and several others are functionally equivalent ${ }^{3}$. Disposable respirators meeting these standards are currently the most commonly used devices in healthcare settings for protecting HCWs during high-risk procedures. Despite significant increases in production capacity since the onset of the COVID-19 pandemic, demand for these devices continues to outstrip supply in most jurisdictions ${ }^{4}$.

Many centers have deployed reusable silicone respirators developed for industrial applications. These masks offer comparable or superior filtration properties for the wearer and can be decontaminated with relative ease. In addition, they have been designed to be worn for longer periods of time than disposable masks. Their reusability makes it possible to predict demand with greater certainty, facilitating planning and ensuring reliable supply once obtained. These masks have two major limitations: their supply is still significantly limited in many areas and they do not filter expired gases. This can be partially mitigated by placing a surgical mask overtop of the reusable respirator, but this can complicate the doffing process potentially increasing the risk of contamination.

Low-cost manufacturing technologies such as 3D printing and molding permit effective small to medium scale local manufacturing of increasingly complex devices using a rapidly growing range of materials. Availability of a validated open-source device designed specifically for healthcare, with well-defined manufacturing and quality control processes that conform to technical standards and are consistent with regulatory requirements can provide a valuable alternative for many centers during the current and future pandemics. Distributed manufacture of such devices can enhance the resilience of supply chains. Numerous respirator designs have been proposed since the start of the current pandemic, but few have been systematically tested in HCWs. There is currently no validated open-source respirator design for healthcare settings.

Respirator performance relies on two crucial factors: the seal of the mask on the user's face which ideally forces all flow to pass through the filter surface, and the filter's performance. Given the availability of ventilator heat-moisture exchange (HME) filters with adequate filtration properties, we focused on developing a well-fitting reusable silicone half-face respirator with a standard $22 \mathrm{~mm}$ port that would connect to a HME filter. Quantitative fit testing in volunteer HCWs showed that a model, with a single pleated-membrane HME filter (Hydro-Guard Mini ${ }^{\mathrm{TM}}$, Intersurgical, Wokingham, UK) exceeded the filtration efficiency of the existing disposable N95 respirators ${ }^{5}$. However, evaluation of this device raised concerns regarding work of breathing, communication, and comfort. The universal one-size-fits-all model did not provide adequate face seal on all users. In this paper, we describe the next major iteration of the design, named "Duo" which now includes separate, valved, inspiratory and expiratory pathways and accommodates up to two filters. The separation of the inspiratory and expiratory paths and addition of the second filter reduces inspiratory resistance, reduces respiratory dead space, and prolongs filter life. The filtration performance of the mask was compared to individually fitted disposable N95s using QNFT in a cohort of $41 \mathrm{HCWs}$, during resting and active maneuvers, per CSA Z94.4-186.

\section{Results}

\section{Device design}

The Duo builds on the previous Simple Silicone Mask (SSM) ${ }^{5}$ and was designed, modified, and produced locally using 3D printing and silicone molding at a hospital laboratory-based workshop (Lynn and Arnold Irwin Advanced Perioperative Imaging Lab, Toronto General Hospital). The design of the Duo focused on 
resolving two main issues from the SSM: improving the face seal and reducing the work of breathing. To improve the face seal the Duo was designed in two sizes (regular and small). To reduce work of breathing, inspiratory resistance and mask dead space had to be reduced. This was accomplished by 1) the introduction of valves to partially separate the inspiratory and expiratory paths, which removed the filter volume from the effective dead space; and 2) addition of a second filtered inspiratory port to reduce resistance. Two Hydro-Guard Mini ${ }^{\mathrm{TM}}$ HME filters (Intersurgical, Wokingham, UK) were used on the inspiratory ports. A single layer of Halyard 100 surgical sterilization wrap (O\&M Halyard Inc., Alpharetta, GA) was used to cover the circular, $26.6 \mathrm{~mm}$ diameter expiratory port (cross sectional area $\sim 5.6 \mathrm{~cm}^{2}$ ).

\section{Device manufacturing}

Computer-aided design of the main mask body and corresponding mold were performed using Onshape.com, v.1.116 (Onshape, Cambridge MA). The 4-part mold was 3-D printed using a Prusa 3 MK3S (Prusa Research, Prague, Czech Republic) and is shown in Figure 1a. A skin safe curable rubber silicone (Dragon Skin 20 ${ }^{\mathrm{TM}}$ 2-part liquid rubber, Smooth-On Inc. Macungie, PA) was poured into the mold and allowed to cure for 3 hours.
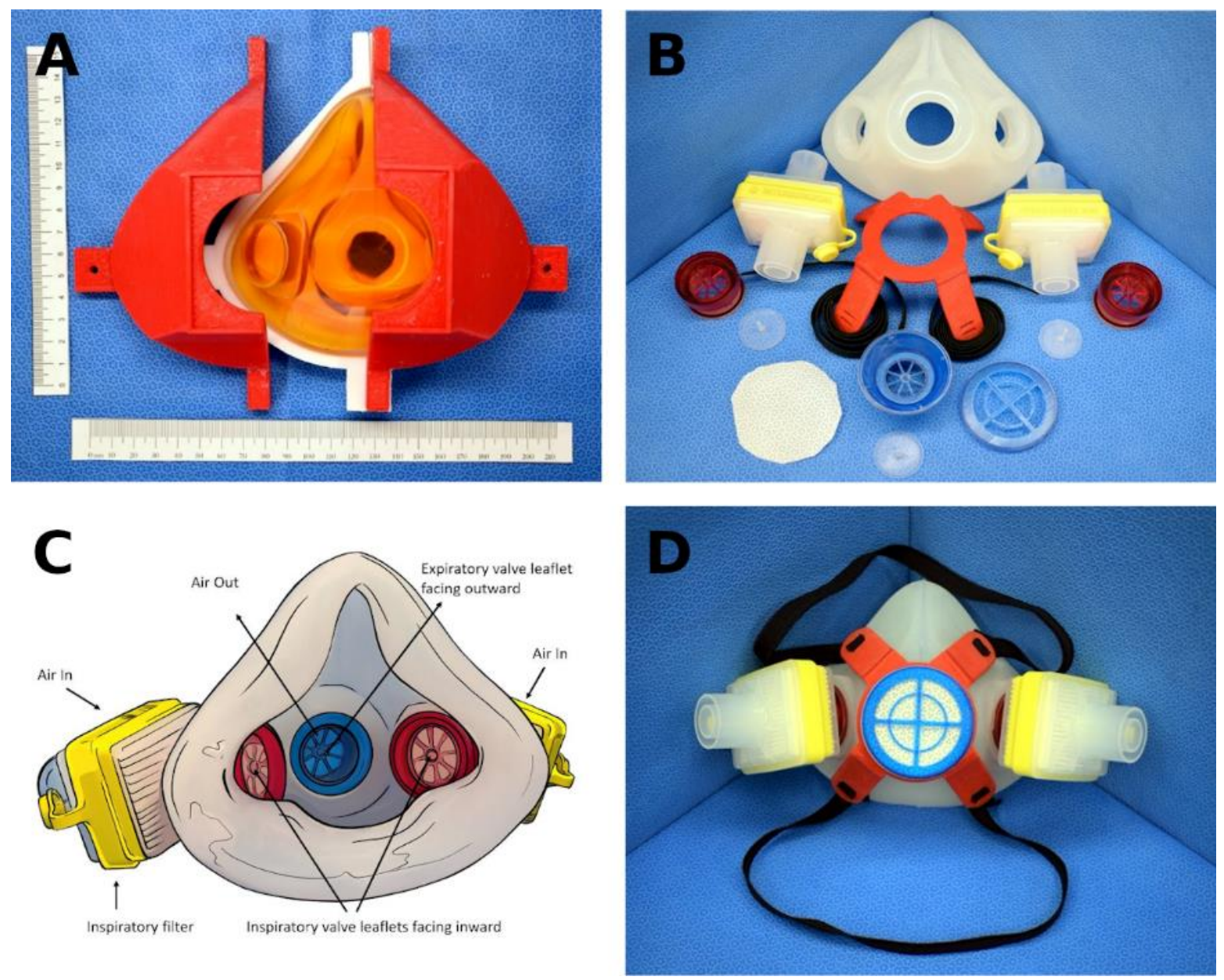

Figure 1. Production process of the Duo silicone respirator. A) The 4-part 3D-printed mold B) Complete set of components C) Valve assemblies D) Assembled Duo respirator.

The three ports in the body (center expiratory port, diameter $26.6 \mathrm{~mm}$, and two inspiratory side ports, diameter $26.3 \mathrm{~mm}$ ) were designed to accept valve seats, printed using a Stratasys $\mathrm{J} 750$ printer (Stratasys Ltd., Eden Prairie MN). The large outer rim of the expiratory valve seat accepts a single $(5 \mathrm{~cm} \times 5 \mathrm{~cm})$ sheet of Halyard 100 surgical sterilization wrap. This is held in place by a removable cap that snaps to the 
valve seat. This configuration filters expired air, similar to a surgical mask. Medical grade silicone mushroom valve leaflets (TME Inc., Toronto, ON) were placed in the valve seats and oriented to ensure unidirectional inspiratory and expiratory flows (Figure 1c). As mentioned above, two pleated-membrane HME filters were placed at the inspiratory ports. A 4-pronged harness was designed, and 3D printed, accommodating two elastic straps $(60 \mathrm{~cm} \times 1 \mathrm{~cm})$.

All components are illustrated in Figure 1b. The fully assembled respirator (weighting 156 grams) is shown in Figure 1d. Complete designs, manufacturing instructions and detailed testing data are available at: https://github.com/tgh-apil/Reusable-N95-Respirator

Figure 1 depicts the mold, disassembled components, and finished device. The complete manufacturing process is outlined in the Supplementary Methods file. All project documentation, including detailed design and manufacturing files, as well as test data, are available at the open-source project repository: https://github.com/tgh-apil/Reusable-N95-Respirator.

\section{Cohort Characteristics}

$41 \mathrm{HCWs}$ (mean [SD] age 36 [7] years; 51\% male) provided informed consent and underwent quantitative fit testing of their hospital approved, qualitative fit-tested (denatonium benzoate), disposable N95 model and the appropriately sized Duo mask. Participant characteristics are described in Table 1. Mean BMI was $22.9[3.4] \mathrm{kg} / \mathrm{m}^{2} ; 80 \%$ of participants had BMls in the normal range $(18.5-24.9) \mathrm{kg} / \mathrm{m}^{2}$. To guide mask fit testing, NIOSH provides a classification of 10 face morphologies, known as panels ${ }^{7}$. Our sample included at least one participant for nine of the ten NIOSH panels. Seven participants $(17.1 \%)$ demonstrated measurements outside of the standard NIOSH panel range. The distribution of N95 models in the cohort was as follows (all models from 3M ${ }^{\mathrm{TM}}$, St. Paul, MN): 1870+ (43.9\%), 1860 (22.0\%), 1860S (17.1\%), $8210(14.6 \%)$, and $9105 \mathrm{~S}(2.4 \%)$.

\section{Quantitative Fit-Testing and Inhalation Resistance Testing}

Quantitative fit test as prescribed by CSA Z94.4-18 consists of 7 trials: normal breathing, deep breathing, turning, nodding, talking, bending, and normal breathing repeated. An overall pass requires passing at least 6 of 7 trials. The overall pass rate of QNFT fitted disposable N95s was $58.5 \%(24 / 41)$, and $100 \%$ for the Duo. Table 1 presents the distribution of participant demographics, anthropometric characteristics, and N95 models by the QNFT pass/fail outcome for the disposable N95s. The specific N95 model was a significant predictor of failure $(p=0.016)$. The $1870+$ and 8210 models had the highest pass rates with $14 / 18(78 \%)$ and $5 / 6(83 \%)$ overall respectively.

Table 1. Demographics, anthropometric characteristics and disposable N95 model distributions overall and by disposable N95 quantitative fit-test outcome. Percentage values are based on column totals.

\begin{tabular}{|c|c|c|c|c|}
\hline & \multirow{2}{*}{$\begin{array}{c}\text { Total / } \\
\text { Overall Value }\end{array}$} & \multicolumn{2}{|c|}{$\begin{array}{c}\text { Disposable N95 Overall } \\
\text { QNFT Result }\end{array}$} & \multirow{2}{*}{ P-Value } \\
\hline & & Pass & Fail & \\
\hline \multicolumn{5}{|l|}{ Demographics } \\
\hline $\mathrm{N}$ & 41 & 24 & 17 & \\
\hline Age, mean (SD), y & $36.1(7.0)$ & $35.7(7.1)$ & $36.6(7.0)$ & 0.68 \\
\hline Female, n (\%) & $20(48.8 \%)$ & $11(45.8 \%)$ & $9(52.9 \%)$ & 0.65 \\
\hline BMI, mean (SD), $\mathrm{kg} / \mathrm{m}^{2}$ & $22.9(3.3)$ & $22.8(3.5)$ & $22.9(3.3)$ & 0.98 \\
\hline Normal weight (18.5-24.9) & $33(80.5 \%)$ & $21(87.5 \%)$ & $12(70.6 \%)$ & \multirow[t]{2}{*}{0.047} \\
\hline Overweight (25-30) & $6(14.6 \%)$ & $1(4.2 \%)$ & 5 (29.4\%) & \\
\hline
\end{tabular}




\begin{tabular}{|c|c|c|c|c|}
\hline Obese $(>30)$ & $2(4.9 \%)$ & $2(8.3 \%)$ & 0 & \\
\hline \multicolumn{5}{|l|}{ Anthropometric variables } \\
\hline Face width, mean (SD), mm & $133(12)$ & $132(9)$ & $134(15)$ & 0.34 \\
\hline Face length, mean (SD), mm & $120(9)$ & $120(7)$ & $121(12)$ & 0.77 \\
\hline \multicolumn{5}{|l|}{ NIOSH panel, n (\%) } \\
\hline 1 & $2(4.9 \%)$ & $2(100 \%)$ & 0 & \multirow[t]{11}{*}{0.21} \\
\hline 2 & $0(0.0 \%)$ & - & - & \\
\hline 3 & $7(17.1 \%)$ & $5(71.4 \%)$ & $2(28.6 \%)$ & \\
\hline 4 & $4(9.8 \%)$ & $2(50.0 \%)$ & $2(50.0 \%)$ & \\
\hline 5 & $3(7.3 \%)$ & $2(66.7 \%)$ & $1(33.3 \%)$ & \\
\hline 6 & $5(12.2 \%)$ & $5(100 \%)$ & 0 & \\
\hline 7 & $8(19.5 \%)$ & $4(50.0 \%)$ & $4(50.0 \%)$ & \\
\hline 8 & $1(2.4 \%)$ & $1(100 \%)$ & 0 & \\
\hline 9 & $2(4.9 \%)$ & $1(50.0 \%)$ & $1(50.0 \%)$ & \\
\hline 10 & $2(4.9 \%)$ & 0 & $2(100 \%)$ & \\
\hline NA & $7(17.1 \%)$ & $2(28.6 \%)$ & $5(71.4 \%)$ & \\
\hline \multicolumn{5}{|l|}{ Disposable N95 model n (\%) } \\
\hline $1870+$ & $18(43.9 \%)$ & $14(77.8 \%)$ & $4(32.2 \%)$ & \multirow[t]{5}{*}{0.016} \\
\hline 1860 & $9(21.9 \%)$ & $2(32.2 \%)$ & $7(77.8 \%)$ & \\
\hline $1860 S$ & $7(17.1 \%)$ & $3(42.9 \%)$ & $4(57.1 \%)$ & \\
\hline 8210 & $6(14.6 \%)$ & $5(83.3 \%)$ & $1(16.7 \%)$ & \\
\hline $9105 S$ & $1(2.4 \%)$ & 0 & $1(100 \%)$ & \\
\hline
\end{tabular}


Pass rates and fit factors for individual maneuvers are presented in Table 2 and Figure 2. All participants passed all individual trials with the Duo. The median overall fit-factors were 2947 and 77.2 for the Duo and disposable N95 respirators respectively. Fit factors were consistently higher with the Duo across all individual maneuvers (Table 2 and Figure 3; $p<0.001$ by Wilcoxon signed-rank test). The lowest pass rate with the disposable N95s was during speech: 20/41 (48.8\%). The disposable N95 pass rate was lower during dynamic maneuvers (turning, nodding, talking, bending; 100/164, 61\%) than stationary maneuvers $(89 / 123,73 \%)$. (Figure 4; $p<0.0001$ by two-sample test of proportions)

Table 2. Comparison of pass rates and fit factors across the 7 maneuvers in the QNFT. P-values are based on comparison of harmonic means using Wilcoxon Signed-rank Test.

\begin{tabular}{|c|c|c|c|c|c|}
\hline & \multicolumn{2}{|c|}{ Disposable N95 } & \multicolumn{2}{|c|}{ Duo } & \multirow[b]{2}{*}{ P-value* } \\
\hline & $\begin{array}{c}\text { Fit- Factor } \\
\text { Harmonic } \\
\text { Mean } \\
{[95 \% \mathrm{Cl}]}\end{array}$ & $\begin{array}{l}\text { Pass } \\
\text { N (\%) }\end{array}$ & $\begin{array}{c}\text { Fit-Factor } \\
\text { Harmonic } \\
\text { Mean } \\
{[95 \% \mathrm{Cl}]}\end{array}$ & $\begin{array}{l}\text { Pass } \\
\text { N (\%) }\end{array}$ & \\
\hline Normal Breathing & $\begin{array}{l}86.7 \\
{[54.7-208.0]}\end{array}$ & 27 (65.9\%) & $\begin{array}{l}3936 \\
\text { [2959- 5873] }\end{array}$ & $41(100 \%)$ & $P<0.0001$ \\
\hline Deep Breathing & $\begin{array}{l}69.5 \\
{[41.9-202.3]}\end{array}$ & $22(53.7 \%)$ & $\begin{array}{l}4752 \\
{[3619-6915]}\end{array}$ & $41(100 \%)$ & $P<0.0001$ \\
\hline Head Side-to-side & $\begin{array}{l}80.1 \\
{[50.9-188.7]}\end{array}$ & $28(68.3 \%)$ & $\begin{array}{l}4589 \\
{[3432-6923]}\end{array}$ & $41(100 \%)$ & $P<0.0001$ \\
\hline $\begin{array}{l}\text { Head Up-and- } \\
\text { Down }\end{array}$ & $\begin{array}{l}79.0 \\
{[52.6-} \\
158.8]\end{array}$ & $28(68.3 \%)$ & $\begin{array}{l}4432 \\
{[3288-6797]}\end{array}$ & $41(100 \%)$ & $P<0.0001$ \\
\hline Talking out loud & $\begin{array}{l}73.8 \\
{[57.3-103.7]}\end{array}$ & $21(51.2 \%)$ & $\begin{array}{l}1798 \\
{[1524-2192]}\end{array}$ & $41(100 \%)$ & $P<0.0001$ \\
\hline Bending over & $\begin{array}{l}62.1 \\
{[42.0-119.2]}\end{array}$ & $23(56.1 \%)$ & $\begin{array}{l}2312 \\
{[1353-7949]}\end{array}$ & $41(100 \%)$ & $P<0.0001$ \\
\hline Normal Breathing & $\begin{array}{l}100.2 \\
{[65.9-208.2]}\end{array}$ & $30(73.2 \%)$ & $\begin{array}{l}2087 \\
{[1165-} \\
10013]\end{array}$ & $41(100 \%)$ & $P<0.0001$ \\
\hline Overall & $\begin{array}{l}77.4 \text { [51.9- } \\
152.1]\end{array}$ & $25(61.0 \%)$ & $\begin{array}{l}2959 \text { [2228- } \\
4405]\end{array}$ & $41(100 \%)$ & $P<0.0001$ \\
\hline
\end{tabular}




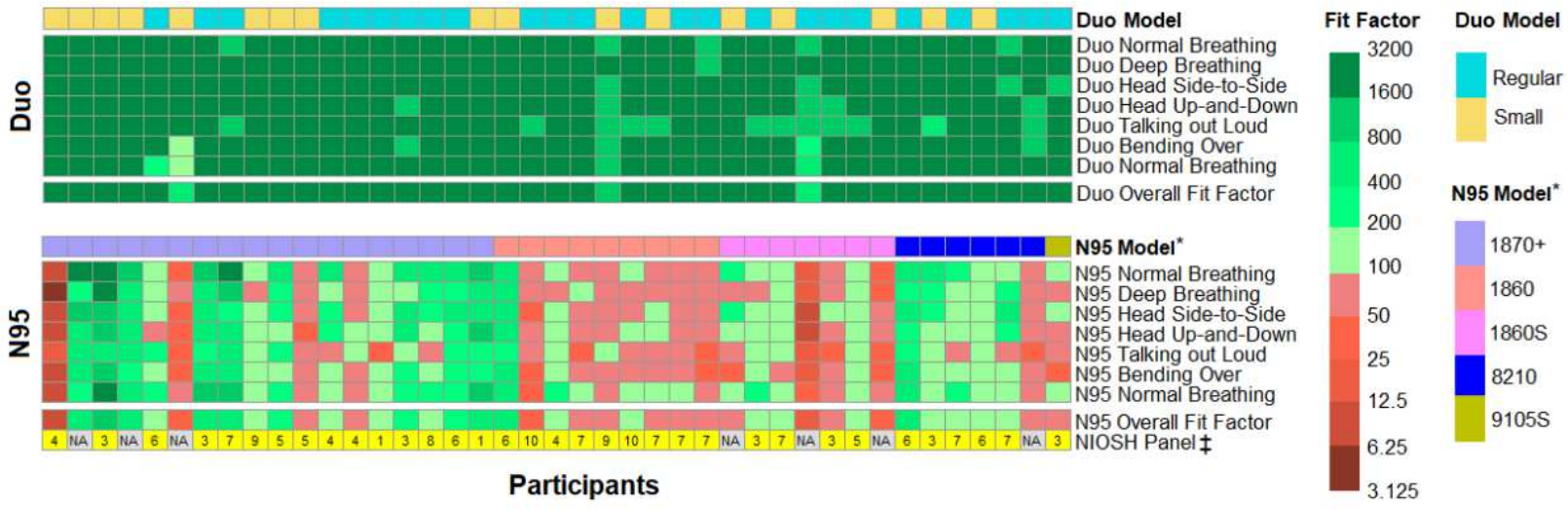

Figure 2. Heat maps illustrating the results of quantitative fit testing (QNFT) for the Duo and disposable N95 masks. The results for each participant are represented along each column with the Duo on top and Disposable 95 at bottom. Rows represent the fit factors for each of the 7 maneuvers, overall fit factor, mask models (legends on left) as well as NIOSH morphometric panel number (bottom row). Participants have been ordered by their qualitatively fit-tested disposable N95 models. Fit factor values are colour coded with green values representing fit factors $>100$, indicating a pass according to CSA Z94.4-18 and red boxes indicating failures. 


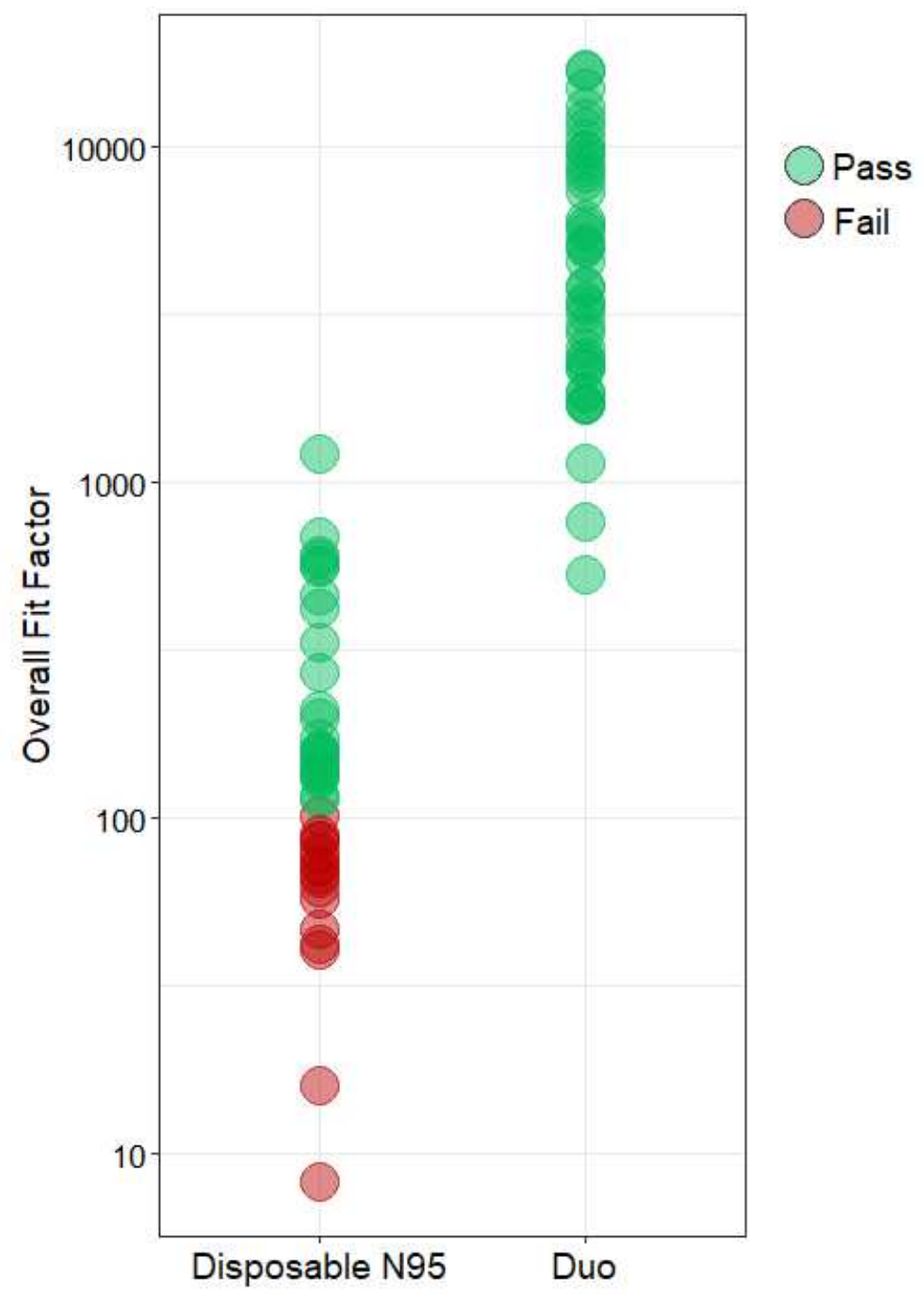

Figure 3. Comparison of overall fit factors for disposable N95 vs the Duo in 41 health care workers. Green values indicate a pass according to CSA Z94.4-18. Log scale. $p<0.001$ (Wilcoxon signed-rank test). 


\section{Pass Rates for Disposable N95 and Duo Across Dynamic and Stationary Maneuvers}

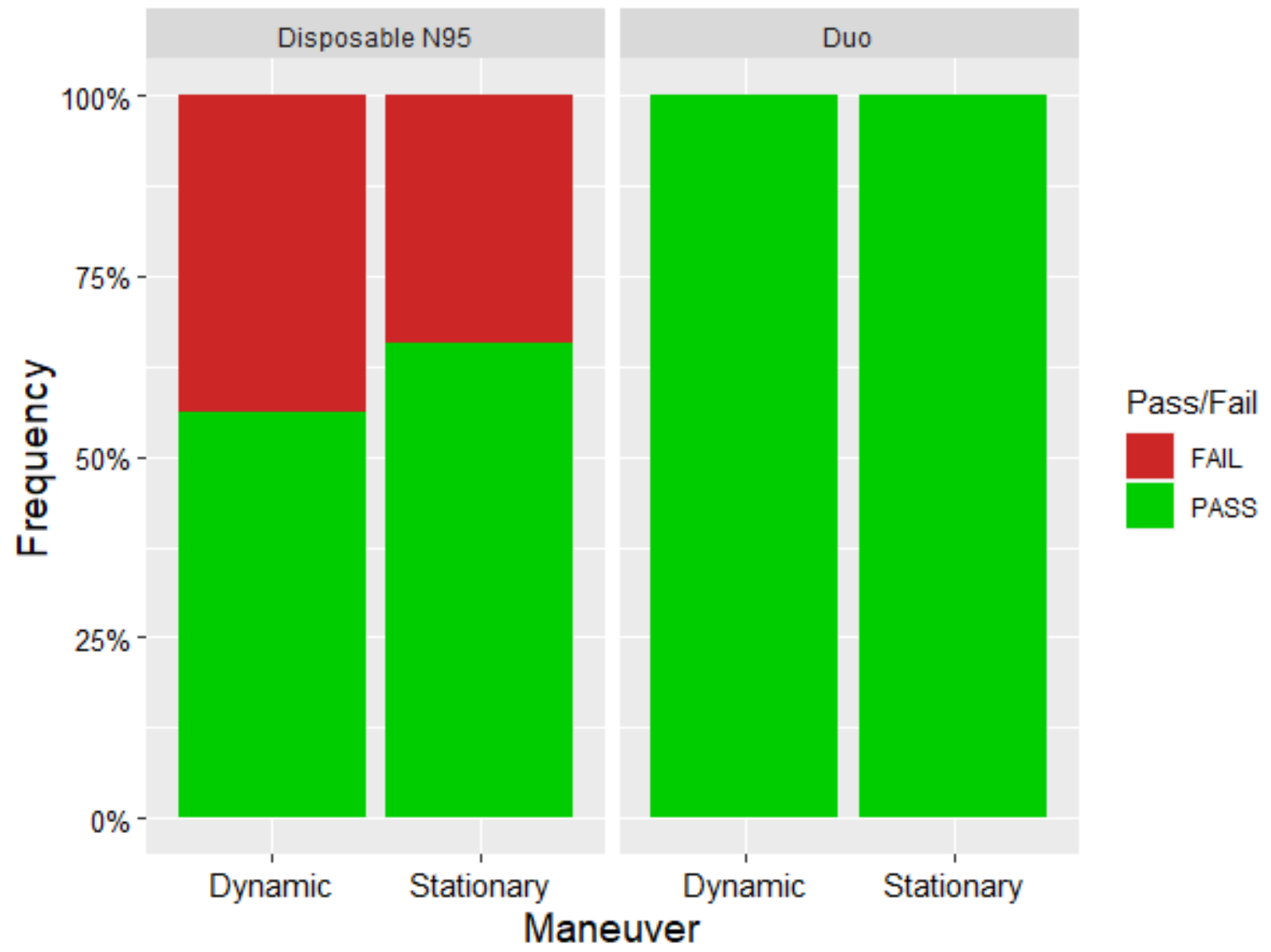

Figure 4. Comparison of individual maneuver fit factors for disposable N95 vs the Duo in 41 health care workers, aggregated by stationary (normal breathing, deep breathing) and dynamic maneuvers (turning, nodding, speaking, bending). $p<0.0001$ by two-sample test of proportions.

Results for inspiratory resistance are shown Table 3. NIOSH, requires that the resistance of nonpowered, air-purifying particulate respirators shall not exceed $3.5 \mathrm{~cm} \mathrm{H} \mathrm{H}_{2} \mathrm{O}$ at a flow rate of $85 \mathrm{~L} / \mathrm{min}^{8}$. In the single filter condition, the resistance measured was $7.44 \pm 0.11 \mathrm{~cm} \mathrm{H}_{2} \mathrm{O}$ at $80 \mathrm{~L} / \mathrm{min}$. This resistance exceeds the NIOSH limit, and is far higher than the resistance values of the disposables N95 models as reported by a NIOSH study (range 0.7 for $3 \mathrm{M} 1870$ to $1.0 \mathrm{~cm} \mathrm{H}_{2} \mathrm{O}$ for the $3 \mathrm{M} 8000$ ) ${ }^{9}$. However, with two filters, the resistance of the Duo was reduced to $2.47 \pm 0.11 \mathrm{~cm} \mathrm{H} \mathrm{H}_{2} \mathrm{O}$ at $80 \mathrm{~L} / \mathrm{min}$, therefore meeting the $\mathrm{NIOSH}$ requirement.

Table 3. Summary of the average pressure and the average flow (air suction set to $25 \mathrm{~L} / \mathrm{min}, 50 \mathrm{~L} / \mathrm{min}, 75$ $\mathrm{L} / \mathrm{min}$, and $80 \mathrm{~L} / \mathrm{min}$ ). The flow measurements were recorded at the back of the mouth of the headform (Oropharynx).

\begin{tabular}{|l|c|c|}
\hline Test Condition & $\begin{array}{c}\text { Flow (L/min) } \\
\text { Mean [SD] }\end{array}$ & $\begin{array}{c}\text { Pressure (cm H20) } \\
\text { Mean [SD] }\end{array}$ \\
\hline Single Filter & $25.24[0.16]$ & $1.53[0.08]$ \\
\hline
\end{tabular}




\begin{tabular}{|l|c|c|}
\hline Double Filter & $25.33[0.15]$ & $0.41[0.10]$ \\
\hline Single Filter & $49.43[0.18]$ & $3.73[0.11]$ \\
\hline Double Filter & $49.66[0.21]$ & $1.33[0.11]$ \\
\hline Single Filter & $74.63[0.29]$ & $6.72[0.13]$ \\
\hline Double Filter & $74.79[0.31]$ & $2.32[0.12]$ \\
\hline Single Filter & $79.85[0.27]$ & $7.44[0.07]$ \\
\hline Double Filter & $80.01[0.30]$ & $2.47[0.11]$ \\
\hline
\end{tabular}

\section{Discussion}

The surge in demand for $\mathrm{N} 95$ and comparable respirators during the current pandemic has repeatedly exceeded the capacity of global manufacturing and supply chains. This has led to critical shortages even in traditionally well-resourced countries and has exacerbated the chronic shortages that are a constant reality for much of the world's population ${ }^{10}$.

In this paper we describe the development and quantitative user testing of a reusable, valved, silicone respirator that can be manufactured locally with low-cost infrastructure, and exceeds the filtration performance of the current standard of care for high-risk procedures. In addition, we performed preliminary testing of flow resistance. The device requires further refinement and testing to rigorously evaluate these and other factors effecting user performance such as heat and moisture retention, and voice transmission. User comfort is a critical determinant of both user performance and compliance. Several survey studies have shown that HCWs are only partly compliant with guidelines for respiratory protection often citing lack of device comfort as a dominant factor ${ }^{11-13}$.

Our test results join the growing body of evidence on the poor performance of disposable N95 respirators. The overall QNFT pass rate of $58.5 \%$ found in our study is slightly lower but generally in keeping with previously reported pass rates of $70-83 \%^{14-16}$. In our sample we noted an effect of the disposable N95 model, with the recently introduced (and currently in very short supply) $3 \mathrm{M}^{\mathrm{TM}} 1870+$ and 8210 having markedly better performance than other models.

Few studies have assessed novel respirator performance. Most rely on user seal checks to detect mask leaks and a few have used qualitative fit testing with denatonium benzoate (Bitrex® QLFT) ${ }^{17-19}$. Our study used an objective and standardized path for the evaluation of reusable face-masks using the same QNFT procedures applied to an earlier version of the device 5 . As in the previous study, we observed a clinically significant difference in efficiency between the disposable N95 and the Duo respirators, with overall fit factors of 77.2 and 2947 respectively. The improved fit factor of the Duo may be explained by the improved face seal provided by silicone compared to polypropylene fibers in the disposable N95 models. To be noticed, we observed a difference in total filter surface area between the Duo $438.6 \mathrm{~cm}^{2}$ versus 135 $\mathrm{cm}^{2}$ (1804S) - $182 \mathrm{~cm} 2(1870+)$ for the disposable N95 models, however this difference does not explain the difference in resistance of the two respirators. At $80 \mathrm{~L} / \mathrm{min}$ of inspiratory flow, the inhalation resistance (maximum pressure drop) in the Duo was $2.47 \mathrm{~cm} \mathrm{H}_{2} \mathrm{O}$ while the resistance of disposable respirator ranged from 0.7 for $3 \mathrm{M} 1870$ to $1.0 \mathrm{~cm} \mathrm{H}_{2} \mathrm{O}$ for the $3 \mathrm{M} 8000$. With resistance less than $3.5 \mathrm{~cm} \mathrm{H}_{2} \mathrm{O}$ at 80 $\mathrm{L} /$ min Duo appears to meet the NIOSH requirements ${ }^{3}$ Reusable valved silicone respirators have been proposed as an alternative to disposable N95s and industrial versions such as the $3 \mathrm{M} 7500\left(3 \mathrm{M}^{\mathrm{TM}}\right.$, St. Paul, MN) have been adopted by many centers ${ }^{20}$. A significant concern with these respirators in health care settings is the potential for aerosol spread through unfiltered expired air ${ }^{21}$. Previous studies have 
also identified increased temperature and work of breathing as the principal reasons for inappropriate use of respirators in general ${ }^{22,23}$. Functional dead space volume, flow resistance and thermal properties result in subjective discomfort characterized as increased breathing resistance, fatigue, drowsiness, heat, and moisture build-up within the mask. These are in turn associated with physiological signs of stress such as increased heart rate ${ }^{16,24,25}$.

In our candidate device, the separation of inspiratory and expiratory paths using valves; the addition of a second inspiratory port and filter; and the addition of an expiratory filter material using Halyard sterilization wrap, $\mathrm{H} 100$ (O\&M Halyard Inc., Alpharetta, GA) were intended to mitigate these problems. The use of the sterilization wrap as expiratory filter material is appropriate since they are readily available in the hospital setting. Moreover, the Halyard $(\mathrm{H} 100)$ sterilization wrap has been shown to have a particle filtration efficiency (PFE 79.8\%) comparable to the standard hospital surgical mask (PFE 74.4\%) ${ }^{26}$. The unidirectional air flow reduces moisture build-up and contamination of the inspiratory filters potentially prolonging their life. Moisture can degrade the performance of electrostatic filter materials and raises concerns of microbial and fungal growth with prolonged use ${ }^{27}$. Therefore, the Duo provides a more ergonomic device that can be tolerated for longer periods with fewer breaches in compliance and impairment of performance. In response to feedback on the first design iteration, the current version of the respirator was designed in two sizes, with a new smaller version for wearers with smaller nasal bridges. This model also provides better accommodation for eyeglasses.

Unfortunately, our preliminary testing suggests that despite the unidirectional flow, the effective dead space of the device may lead to unacceptable levels of rebreathing and carbon dioxide retention (above $2 \% \mathrm{CO}_{2}$ retention $)^{28}$. Further testing and a revision of the mask geometry to reduce internal volume are therefore indicated before the device can meet all technical requirements for $\mathrm{CO}_{2}$ retention as required by $\mathrm{NIOSH}^{28}$.

The Duo requires further testing and likely revisions addressing issues such as exhalation flow resistance, $\mathrm{CO}_{2}$ retention and speech transmission. The major limitation of our study population was the low number of obese and overweight participants (<10 participants), and that not all NIOSH bivariate facial panel sizes were represented ${ }^{7}$. The HCW population may be less obese than the general population, however this warrants a larger study sample size from other frontline worker groups. Our current experience indicates the smaller and very wide faces would require special respirator sizing and shaping. This problem applies equally to disposable N95 respirators that have failed subjective negativepressure fit-check and QNFT.

The failures of global manufacturing and supply systems during this pandemic are multi-factorial. One recurrent theme is centralization, with a small number of producers responsible for the bulk of global supply for many critical items. This centralization bottlenecks efforts to ramp up production through a small (not infrequently a single) organization. Distributed manufacturing using lower-cost and more flexible infrastructure such as 3D printing presents a tantalizing possibility for addressing this challenge. Traditionally used for prototyping, such systems are increasingly used for small to medium scale production, including Class 1 medical devices such as stethoscopes and face shields ${ }^{29,30}$ and are being actively explored for more complex processes such as biochemical manufacturing ${ }^{31}$.

Despite the promise and the significant boost provided by the pandemic, distributed manufacturing of regulatory approved medical devices is still in early stages and faces numerous challenges. Distributed manufacturing relies fundamentally on open-source intellectual property licensing that allows a network of manufacturers to collaboratively build capacity and share resources. Building and sustaining such collaborations in the face of the harshly competitive dynamics of global markets is a significant challenge. Precedents for such collaboration however do exist and have been fundamental to the development of our global digital infrastructure, to cite one example. During the current pandemic, we have seen numerous open-source collaborations aiming to address global shortages of medical supplies ranging from ventilators to PPE. To date, a wide number of designs have been developed and shared, but very few have reached even early levels of field testing, not to mention regulatory evaluation and the development of viable product life cycle plans for decentralized manufacturing, quality control, 
distribution, post deployment surveillance, device decontamination and reprocessing, decommissioning and disposal.

Along with further device testing, translation of devices such as the Duo into marketable products requires the development of streamlined manufacturing, quality control and other product life cycle processes in keeping with a risk management framework as outlined in standards such as ISO $14971^{32,33}$. Such risk management frameworks are increasingly central to all levels of device development, manufacturing processes and regulation, including the very development of technical standards and regulatory requirements themselves. If carried out successfully, devices like the Duo can be manufactured in-house, at most hospitals, at reasonable cost, with initial infrastructure investments on the order of CAD \$10 20,000. Many large hospitals, especially academic research centers, already have the necessary administrative and technical infrastructure required for managing production and quality control processes and to obtain certifications required for the manufacturing of medical devices (for example ISO 13485). This combination of hospital-based infrastructure and structured device development process in an academic setting outlines one potentially viable path for translating the promise of open-source hardware development and distributed manufacturing into the frontlines of patient care.

\section{Methods}

\section{Device design and manufacturing}

The Duo was designed, and locally manufactured using 3D printing and silicone molding at a hospital laboratory-based workshop. Complete designs, manufacturing instructions and detailed testing data are available at: https://github.com/tgh-apil/Reusable-N95-Respirator.

\section{Decontamination}

As per CDC instructions, possible methods for decontamination of reusable respirators are household bleach, vaporous hydrogen peroxide, ultraviolet germicidal irradiation, and moist heat. All reusable components of the respirator were sanitized prior to and after each participant. A diluted household bleach solution (5.25\% sodium hypochlorite) was utilized as recommended by the CDC and outlined in CSA Z94.4-2018 ${ }^{6}$. To prepare the cleaning solution, approximately $\sim 180 \mathrm{~mL}$ (3/4 cup) of household bleach was added to $3.8 \mathrm{~L}$ of cold water. The respirator components were divided and remained submerged in the solution for 5 minutes, then rinsed thoroughly with tap water. After rinsing, the components were airdried in a well-ventilated and clean area prior to use. HME filters were not reused. The reusability of the silicone respirator body, the harness and elastic straps were tested by disinfecting each component 30 times consecutively, with preserved mass and integrity.

\section{Quantitative Fit-Testing}

To test the filtration performance of the Duo respirator, a prospective observational cross-sectional study was conducted between May and June 2020 in accordance with the Declaration of Helsinki and Good Clinical Practice guidelines. The study was approved by the University Health Network (UHN) Research Ethics Board (\#20-5435). HCWs were recruited Toronto General Hospital and written consent was obtained from each participant. Participants were required to have passed the UHN Occupational Health and Safety qualitative fit test on a disposable N95 respirator within the previous 12 months. Participants were excluded if they reported having flu-like symptoms or had facial hair.

Respirators were tested on 41 healthcare workers, using QNFT on the AccuFIT 9000 ${ }^{\mathrm{TM}}$ (Levitt-Safety, Oakville, Canada) respirator fit tester (CSA Z94.4-18 protocol). SARS-CoV-2 virions have been reported in the range of $50-200 \mathrm{~nm}$ in diameter ${ }^{34}$. In a recent aerosol protection study in reusable respirators, Sickbert-Bennett et al. highlighted that the US Code of Regulations (42 CFR Part 84, subpart K) stipulates the median particle size of $75 \mathrm{~nm}+/-20 \mathrm{~nm}$ as the range for testing and certification of N95 respirators ${ }^{35}$. They also argue that protection against aerosols of $50 \mathrm{~nm}$ implies at least similar protection against much larger aerosols and droplets (greater than $3 \mathrm{um}$ ), because of the dynamics of aerosol movement by deposition, diffusion (Brownian motion), impaction and sedimentation. The AccuFIT ${ }^{\mathrm{TM}}$ 
system detects particles of range $20 \mathrm{~nm}$ to $1 \mathrm{um}$. During the test $\mathrm{NaCl}$ is aerosolized into the testing room. The testing device samples ambient air and air within the mask using two different channels and compares aerosol particle count values to compute a fit factor, as the ratio of two particle counts (ambient count being the numerator).

For each participant tested, demographic data was recorded including age, gender, height, and weight. Anthropomorphic measurements of face-width (bizygomatic breadth) and face length (menton-sellion length) was also measured and recorded. These measurements have been found to significantly correlate with fit. National Institute for Occupational Safety and Health (NIOSH) defines a panel of 10 facial morphology categories based on these measurements, which are used in testing of industrial respirators ${ }^{7}$. Face width is defined as the maximal horizontal distance as measured between the zygomatic arches. Face length is defined as the distance from the prominence of the nasal bridge to the prominence of the chin measured in the mid-sagittal plane. A fresh disposable N95 of the model for which the user was approved by QLFT was modified by the insertion of a standard testing grommet which was connected to the sampling line of the test device. Participants were instructed to don the mask and perform positive and negative pressure seal checks prior to testing. Adjustments were made until there was no detectable seal leak by pressure check. The Duo was equipped with a testing port and was also donned and adjusted to ensure no detectable seal leak in a similar fashion.

Fit factor measurements were recorded for each of the seven standard maneuvers according to CSA Z94.4-18: normal breathing, deep breathing, turning head side to side, nodding up and down, counting out loud. The overall fit factor is calculated as the harmonic mean of the fit factors for each of the 7 maneuvers. The pass criteria for an N95 respirator requires both an overall fit factor and at least six of seven individual maneuver fit factors to be 100 or greater ${ }^{6,34}$. The maneuvers were categorized as either stationary (normal breathing, deep breathing) or dynamic (turning, nodding, talking, bending) for further analysis.

\section{Inhalation Resistance Testing}

Inhalation resistance testing was performed by mounting the Duo respirator on an anthropomorphic head form developed in-house. The respirator was fitted to the head form without any leaks. Wall suction was used as the vacuum source, thus creating a continuous inhalation flow through the respirator and into the mouth of the head form. To measure and record flow ( $\mathrm{L} / \mathrm{min})$ and pressure $(\mathrm{cmH} 2 \mathrm{O})$, a FluxMed $\mathrm{GrH}$ (MBMed Company, Buenos Aires Argentina) portable respiratory mechanics monitor was used. Measurements were acquired at the back of the mouth (oropharynx level) of the head form. Testing conditions included the measurement of resistance at various flow rates and with single and double inspiratory filters. Suction was titrated to achieve flows of $25 \mathrm{~L} / \mathrm{min}, 50 \mathrm{~L} / \mathrm{min}, 75 \mathrm{~L} / \mathrm{min}$, and $80 \mathrm{~L} / \mathrm{min}$ and the corresponding pressure and flow readings were recorded using the FluxView software (MBMed Company, Buenos Aires Argentina). In the single filter condition, the second filter port was sealed. Pressure and flow readings were recorded and averaged over 120 seconds at $256 \mathrm{~Hz}$.

\section{Sample Size Calculation}

The minimum sample size of 40 was selected firstly by estimation of the failure rate of less than one percent of the Duo and 33\% of the disposable commercial N95, according to known studies of N95 by QLFT and internal reviews assessing N95 performance ${ }^{15}$. Sample size which was determined by accounting for two groups, an alpha of 0.05 and power of 0.8 , indicated that a minimum of 32 volunteers were required to detect a $20 \%$ difference in overall pass rates. When each of the 7 "run" of the QNFT was treated as an observation, 41 fit tests resulted in $287(7 \times 41)$ paired runs, which was enough to detect a $3 \%$ difference in pass rates of runs with $95 \%$ confidence.

\section{Statistical analysis}

Statistical analysis was executed using Stata 14 (StataCorp. 2015, College Station, TX), and RStudio version 1.3 (RStudio 2020, Boston, MA) for data visualizations. Univariate analysis was performed on demographic and risk factors likely to influence disposable commercial mask fit failure. The nonparametric Wilcoxon signed-rank test was then used to compare differences in fit-factor scores of paired 
runs of equal sample size; the non-parametric Kruskal-Wallis test was also used to compare differences in fit factor scores of different sample sizes. Median overall fit factors were selected as summary statistics to compare the mask performances in the group.

\section{Study limitations}

In this study we present the development and quantitative testing of a reusable silicone respirator that can be locally manufactured using low-cost desktop infrastructure. Using standardized quantitative fit testing in a cohort of $41 \mathrm{HCWs}$, we compared the performance of the mask to the individually fitted disposable N95 masks. Nevertheless, this work has a number of limitations. First is the low number of obese and overweight participants, and the absence of some of the NIOSH bivariate facial panel sizes in our population ${ }^{7}$. An increase of the sample size may allow us to represent the HCW population of different facial type ranges and body size.

Secondly, our testing had some deviations from the $\mathrm{NIOSH}$ requirements for the determination of inhalation resistance for air-purifying respirators ${ }^{8}$. Our vacuum source was only capable of providing 80 $\mathrm{L} / \mathrm{min}$ of continuous suction, and not $85 \mathrm{~L} / \mathrm{min}$ as required by the $\mathrm{NIOSH}$ testing procedure ${ }^{8}$. The measurements were recorded at the back of the mouth of the head form, and not at the mouth. This is because we could not fit the sensor near the mouth without compromising the seal of the respirator to the headform. However, both the European and NIOSH standards for resistance testing are unclear with regards to the specific site of measurement during inhalation resistance testing ${ }^{8,36}$. Finally, NIOSH recommends the use of the Setra Datum 2000 Model 239 digital manometer to measure resistance, and the use of the Brooks Instrument Co. Model 5853S Mass Flow Controller with Brooks Control and Readout Unit Model $0154^{8}$. We did not have access to the $\mathrm{NIOSH}$ specified equipment and used the FluxMed $\mathrm{GrH}$ respiratory mechanics monitoring system to measure resistance and flow.

\section{Disclaimer}

Respiratory protection devices including half-face elastomeric respirators for use in a workplace setting, including the healthcare setting, must have approval from appropriate regulatory agencies, viz. $\mathrm{NIOSH}$ certification as per Ontario Reg 185/19 sec.10(1) under the Occupational Health and Safety Act ${ }^{37}$.

Therefore, The Lynn and Arnold Irwin Advanced Perioperative Imaging Lab (APIL) and its affiliates do not endorse the use of the stopgap respirators described above until additional testing and regulatory approval have been obtained. This half-face respirator as described is an investigational device under development and has not passed all relevant tests for safety and effectiveness and does not currently meet all regulatory requirements for respirators in Canada. 


\section{References}

1. Siegel, J. D., Rhinehart, E., Jackson, M. \& Chiarello, L. 2007 Guideline for Isolation Precautions: Preventing Transmission of Infectious Agents in Health Care Settings. Am. J. Infect. Contro/ 35, S65S164 (2007).

2. Brosseau, L. \& Ann, R. B. N95 Respirators and Surgical Masks. NIOSH Science Blog https://blogs.cdc.gov/niosh-science-blog/2009/10/14/n95/ (2009).

3. 3M. Comparison of FFP2, KN95, and N95 and Other Filtering Facepiece Respirator Classes. https://multimedia.3m.com/mws/media/17915000/comparison-ffp2-kn95-n95-filtering-facepiecerespirator-classes-tb.pdf (2020).

4. Centers for Disease Control and Prevention. Strategies for Optimizing the Supply of N95 Respirators. https://www.cdc.gov/coronavirus/2019-ncov/hcp/respirators-strategy/crisis-alternate-strategies.html (2020).

5. Ng, W. C. K. et al. Subject validation of reusable N95 stop-gap filtering facepiece respirators in COVID-19 pandemic. PLOS ONE 15, e0242304 (2020).

6. Standards Council of Canada. CAN/CSA-Z94.4-18 Selection, use, and care of respirators. Standards Council of Canada / Conseil canadien des normes https://www.scc.ca/en/standardsdb/standards/29645 (2018).

7. Zhuang, Z., Bradtmiller, B. \& Shaffer, R. E. New Respirator Fit Test Panels Representing the Current U.S. Civilian Work Force. J. Occup. Environ. Hyg. 4, 647-659 (2007).

8. NIOSH. Determination of inhalational resistance test, air-purifying respirators standard testing procedures. Procedure No. TEB-APR-STP-0007. 7 (2019).

9. PPE CASE Reports | NPPTL | NIOSH | CDC. https://www.cdc.gov/niosh/nppt//ppecase.html (2020).

10. McMahon, D. E., Peters, G. A., Ivers, L. C. \& Freeman, E. E. Global resource shortages during COVID-19: Bad news for low-income countries. PLoS Negl. Trop. Dis. 14, e0008412 (2020).

11. Evanoff, B. et al. Compliance with universal precautions among emergency department personnel caring for trauma patients. Ann. Emerg. Med. 33, 160-165 (1999).

12. Gershon, R. R. et al. Compliance with universal precautions among health care workers at three regional hospitals. Am. J. Infect. Control 23, 225-236 (1995). 
13. Kelen, G. D. et al. Adherence to Universal (barrier) Precautions during interventions on critically ill and injured emergency department patients. J. Acquir. Immune Defic. Syndr. 3, 987-994 (1990).

14. Clapham, S. J. Comparison of N95 disposable filtering facepiece fits using bitrex qualitative and TSI Portacount quantitative fit testing. Int. J. Occup. Environ. Health 6, 50-55 (2000).

15. Hon, C.-Y. et al. Comparison of qualitative and quantitative fit-testing results for three commonly used respirators in the healthcare sector. J. Occup. Environ. Hyg. 14, 175-179 (2017).

16. Hayashi, C. \& Tokura, H. The effects of two kinds of mask (with or without exhaust valve) on clothing microclimates inside the mask in participants wearing protective clothing for spraying pesticides. Int. Arch. Occup. Environ. Health 77, 73-78 (2004).

17. Copper 3D. Hack the Pandemic - Copper 3D. NanoHack 2.0 https://copper3d.com/hackthepandemic/ (2020).

18. Kvaternik, D. HEPA Covid Coronavirus Face Mask. Thingiverse https://www.thingiverse.com/thing:4222563 (2020).

19. Alenezi, H., Cam, M. E. \& Edirisinghe, M. A novel reusable anti-COVID-19 transparent face respirator with optimized airflow. Bio-Des. Manuf. (2020) doi:10.1007/s42242-020-00097-1.

20. National Personal Protective Technology Laboratory \& National Institute for Occupational Safety and Health. Stakeholder Meeting on Respiratory Protection for Healthcare Workers. in http://www.cdc.gov/niosh/nppt//resources/certpgmspt/meetings/06182013/healthcareinvitation/ttr06182 013.html (Centers for Disease Control and Prevention, 2013).

21. Centers for Disease Control and Prevention. Interim Infection Prevention and Control Recommendations for Healthcare Personnel During the Coronavirus Disease 2019 (COVID-19) Pandemic. (2020).

22. Shenal, B. V., Radonovich, L. J., Cheng, J., Hodgson, M. \& Bender, B. S. Discomfort and exertion associated with prolonged wear of respiratory protection in a health care setting. J. Occup. Environ. Hyg. 9, 59-64 (2012).

23. Seale, H. et al. "The cookie monster muffler": Perceptions and behaviours of hospital healthcare workers around the use of masks and respirators in the hospital setting. Int. J. Infect. Control 11, (2015). 
24. Lerman, Y., Shefer, A., Epstein, Y. \& Keren, G. External inspiratory resistance of protective respiratory devices: effects on physical performance and respiratory function. Am. J. Ind. Med. 4, 733740 (1983).

25. Li, Y. et al. Effects of wearing N95 and surgical facemasks on heart rate, thermal stress and subjective sensations. Int. Arch. Occup. Environ. Health 78, 501-509 (2005).

26. Long, K. D., Woodburn, E. V., Berg, I. C., Chen, V. \& Scott, W. S. Measurement of filtration efficiencies of healthcare and consumer materials using modified respirator fit tester setup. PLOS ONE 15, e0240499 (2020).

27. Majchrzycka, K., Brochocka, A., Luczak, A. \& Lezak, K. Ergonomics assessment of composite ballistic inserts for bullet- and fragment-proof vests. Int. J. Occup. Saf. Ergon. JOSE 19, 387-96 (2013).

28. Determination of facepiece carbon-dioxide and oxygen concentration levels of tight fitting, powered air-purifying respirators, with the blower unit off or non-powered respirators standard testing procedure (STP). (2020).

29. Pavlosky, A. et al. Validation of an effective, low cost, Free/open access 3D-printed stethoscope. PLOS ONE 13, e0193087 (2018).

30. Wilczynski, J. et al. USING DIGITALLY DISTRIBUTED MANUFACTURING TO ADDRESS CRITICAL NEEDS: America Makes has mobilized the additive manufacturing community to work together to address the nation's medical equipment shortages during the COVID-19 pandemic. (MATERIALS SCIENCE AND CORONAVIRUS SERIES). Advanced Materials \& Processes vol. ISSN: 0882-7958; Volume 178; Issue 722 (2020).

31. NSF Award Search: Award \# 2029249 - EFRI DCheM: Modular SynBio Processing Units for Distributed Manufacturing of High-Value Products. https://nsf.gov/awardsearch/showAward?AWD_ID=2029249.

32. 14:00-17:00. ISO/TR 24971:2020. ISO

https://www.iso.org/cms/render/live/en/sites/isoorg/contents/data/standard/07/44/74437.html. 33. 14:00-17:00. ISO 14971:2019. ISO https://www.iso.org/cms/render/live/en/sites/isoorg/contents/data/standard/07/27/72704.html. 
34. Occupational Safety and Health Administration. Standards - 29 CFR Appendix A to $\S 1910.134$ :

Fit Testing Procedures (Mandatory).

https://www.osha.gov/pls/oshaweb/owadisp.show_document?p_id=9780\&p_table=STANDARDS

(2004).

35. Sickbert-Bennett, E. E. et al. Filtration Efficiency of Hospital Face Mask Alternatives Available for

Use During the COVID-19 Pandemic. JAMA Intern. Med. 180, 1607 (2020).

36. EN 140:1999 Respiratory protective devices - Half masks and quarter masks - Requirements, testing, marking. (1999).

37. Government of Ontario. Occupational Health and Safety Act. R.S.O. 1990, c. O.1 vol. 185/19 (2019).

\section{Acknowledgements}

We thank the Department of Anesthesiology and Pain Management, and the Joint Department of Medical Imaging, at University Health Network for their academic support. In addition, we would like to thank Dr. David Green, from UHN's Techna Institute, for lending space for fit testing; Mr. Quang Nguyen, CEO of TME Inc. for the donation of valve leaflets, materials, and lending Prusa printers; Intersurgical (Burlington, Canada) for their donation of Air-Guard ${ }^{\mathrm{TM}}$ respiratory filters. We would also like to thank Kate Kazlovich for the visual sketches of the Images. Special thanks to Nasa Chau Nguyen (BASc) and Natasha Valenton (BASc) from The School of Applied Science and Engineering, Jessica Trac (BSc) from The MD Program, University of Toronto, for her invaluable work on the designs and revisions of the Duo and its derivatives.

\section{Author contributions statement}

ARMH: Methodology, Investigation, Data curation, Formal analysis, Software, Data Visualization, writing original draft, writing - review \& editing

WCKN: Conceptualization, Funding acquisition, Project Administration, Supervision, Investigation, Methodology, Data curation, Writing - Original Draft, Writing - Review \& Editing

VA: Visualization, Investigation, Writing - Original Draft, Writing - Review \& Editing

MZXX: Data Curation, Formal Analysis, Software, Data Visualization, Writing - Review \& Editing

DR: Writing - Review \& Editing

KK: Visualization, Investigation, Writing - Review \& Editing

EA: Writing - Review \& Editing

$\mathrm{JH}:$ Conceptualization 
AM: Conceptualization, Funding acquisition, Resources, Supervision, Data Visualization, Review \& Editing

All authors provided comments to the various drafts and approved the final version.

\section{Additional information}

\section{Data availability}

All relevant data are within the manuscript and its Supporting information files.

\section{Funding}

This work was supported by operational and infrastructure funding of the Lynn and Arnold Irwin Advanced Perioperative Imaging Lab from the Toronto General and Western Hospital Foundation and salary support for Azad Mashari and William Ng from the Sinai Health Systems-University Health Network Anesthesia Association. The project received funding from The Ontario Centres for Innovation from September 2020 to April 2021. Dmitry Rozenberg receives salary support from the Sandra Faire and Ivan Fecan Professorship in Rehabilitation Medicine.

\section{Competing interests}

Azad Mashari is a pro-bono scientific consultant and research collaborator with Glia Inc (Glia.org), a company affiliated with the London Health Sciences Center, developing open-source medical technologies.

William Ng is a principal investigator with funding from The Ontario Centres for Innovation, with Quang Nguyen (CEO, TME Inc.) as the mandatory industry partner on this fund.

No other authors have conflicts of interests. 


\section{Supplementary Files}

This is a list of supplementary files associated with this preprint. Click to download.

- SupplementaryMethods.docx

- DuoDeidentifiedDataset01.01.2021.xIsx

- DuoDeidentifiedDataset01.01.2021.xlsx

- SupplementaryMethods.docx 